\title{
A Novel Design and Implementation of a 4-DOF Upper Limb Exoskeleton for Stroke Rehabilitation with Active Assistive Control Strategy
}

\author{
Anan Sutapun ${ }^{\mathrm{a}}$ and Viboon Sangveraphunsiri ${ }^{\mathrm{b}, *}$ \\ Department of Mechanical Engineering, Faculty of Engineering, Chulalongkorn University, \\ 254 Phyathai, Patumwan, Bangkok 10330, Thailand \\ E-mail: anansutapun@gmail.com, bviboon.s@chula.ac.th (Corresponding author)
}

\begin{abstract}
We developed a robot, CUREs (Chulalongkorn University Rehabilitation Robotic Exoskeleton system), for upper extremity rehabilitation. The active assistive control strategy based on the impedance force control is developed and implemented to obtain assistive-resistive paths tracking for rehabilitation activities. The desired trajectory or rehabilitated training pattern for each specific patient need to be assigned first by a medical doctor and a physical therapy. The therapist can program the desired trajectory by guiding the patient arm based on the assigned path pattern and the set of via points will be stored and used for generating the desired trajectory. The desired trajectory will be stored specific for the patient and can be called back anytime. During the rehabilitation, the robot can assist and resist the patient's arm to follow the desired trajectory. If the patient has difficulty moving his arm to track the desired path, the robot will help by adding more torque to help the patient to move his arm to reduce the error between the desired path and the actual posture. And if the patient himself can move his arm tracking the desired path, the robot will not apply any more force to assist or resist. The necessary state variables such as angular position and torque can be recorded during the training. The main purpose of the experiment, follow the medical ethic, is to assure that there is no side effect for using this rehabilitation robot. Five subacute stroke patients participated in this pilot study. All patients have severe upper extremity weakness. The medical doctor will assign the training pattern based on patient condition. The result showed that the Fugl-Meyer Assessment Upper Extremity Scale was improved after 10 days of training in all participants without any sign of side effect.
\end{abstract}

Keywords: Upper-limb exoskeleton robot, rehabilitation robot, active assistive control strategy, prerecorded trajectory, stroke.

ENGINEERING JOURNAL Volume 21 Issue 7

Received 1 May 2017

Accepted 5 July 2017

Published 29 December 2017

Online at http://www.engj.org/

DOI:10.4186/ej.2017.21.7.275 


\section{Introduction}

The application of robotic can improve muscular strength and movement in patients with neurological or orthopedic lesions especially stroke rehabilitation. Several studies prove that arm therapy has positive effects on the rehabilitation progress of stroke patients [1-7]. So, the interested in using the robotic device to help rehabilitation therapy is increasing. And because the population of the stroke patient is increasing and the number of therapists is insufficient, affecting the quality of rehabilitation and daily care. Basic neurological research suggests that the intensive and task-specific movement training can improve muscle strength and motor recovery in stroke patient [1,2]. The conventional rehabilitation by the therapist has several limitations such as personnel shortage and fatigue of therapists. The consequence is that the training sessions may not sufficient or shorter than the proper training requirement. Using the rehabilitation robot is preferable in various aspect such as the parameter of the control strategy can be varied as to assist and/or resist the movement of patient arm, more training activities, train characteristic can be set to suit a specific patient, longer training duration if necessary, more training sessions can be provided, and one therapist can manage more patients simultaneously. Moreover, training data for each patient can be stored automatically and used for offline analysis.

We can classify rehabilitation robots into 2 categories [8]: the exoskeleton type and the end effector type [9]. For the end-effector type, the patient's limb is attached to the robot arm only at the end-point or endpoint gripper. So, it is rather difficult to isolate specific movements of each of the patient's limb for each training. This contrast to our exoskeleton type. Its configuration consists of four degrees of freedom, the first three degrees represent the human shoulder motion and the last one is elbow motion. The advantage of this configuration is that we can relate the robot joints motion to the motion of the joints of the patient's limb. [10-12].

For the control strategies using in the rehabilitation robot, several control strategies have been proposed and studied [13]. There are two categories of control strategies that suit for the active device: assistive control strategy and challenge-based control strategy. For active assistive control strategy, or impedance-base strategy, a patient need to move his arm to track the pre-defined trajectory. The robot will not intervene as long as the patient can follow the trajectory by himself, in the meantime, the helping force created by the robot joints will be increased when the patient has some difficulty move his hand. The relation of the helping force and the deviation of actual arm trajectory from the desired trajectory can be modelled. This is the impedancebase control. To help the movement of patients with minimal helping force, the control strategy call "Assistance-as-need" (AAN) have been presented in ref [14-15] and the potential of AAN strategies has been shown in upper-limb training of stroke patients [16-17]. The challenge-based control strategy will make the movement of the participant limb more difficult or challenging. For example, the controller will provide the resistive force or make some constraint during the movement of the patient limb or decrease the helping force if the patient preforms well.

The goal of the rehabilitation is to provoke motor plasticity, improve motor recovery, learn motion movement and/or prevent secondary complications such as muscle atrophy, osteoporosis, and spasticity. Many studies showed that using robotic devices in rehabilitation process on stroke patients has positive effects [3-7]. However, there are a few types of research and developments about using robot for rehabilitation in Thailand.

In this paper, the Chulalongkorn University Rehabilitation Robotic Exoskeleton system (CUREs) [12, 18] was implemented to five subacute stroke patients [19]. The active assistive control strategies using impedance control, and the pre-recorded trajectory during therapist-guided assistance [20, 21] were applied. In this implementation, the therapist has planed the rehabilitation process, explained the device function to the patient, adjusted the robot to patients and defined the desired trajectory. Indirect effect, with the aid of the robot for rehabilitation, most patients feel that they have ability to move their arms along the desired trajectory, this feeling might gain more confident in their abilities to move their arms.

\section{4-DOF Upper Limb Exoskeleton Robot for Stroke Rehabilitation}

The main purpose of a 4-DOF upper limb exoskeleton robot, CUREs, for arm physical therapy of stroke patients. For a high performance of force controllability, the mechanical structure must have small weight and low inertia. The mechanical system is designed based on low friction, no backlash, low inertia, large dynamic range, and back-drivable. The exoskeleton joins are designed to align the human arm joints. There 
are four active joints, actuated by four brushless dc servo motors, use cable-base transmission system that lend to zero backlash and provide safety for the impact force. The maximum of the transmission ratio is 12.5 so the robot has good back-drivability. For the shoulder movement, the rotation axis of joint 1 to joint 3 of the robot are perpendicular and intersect at the patient's shoulder joint. And the elbow movement, joint 4 of the robot will align to the rotation axis of the patient's elbow joint as showed in Fig. 1(a). The link offset of the robot arm can be adjusted to suit of a patient arm. The joint-link coordinate frames were defined and attached to each link for joint-link description. More detail about coordinate assignment can consult in [22, 23]. The forward kinematic was described as detailed in [12]. Each joint variable can be measured from an encoder attached to each joint. Moreover, this robot has a smooth motion that showed in the study of the mobility. More detail about the mobility of this exoskeleton can consult more in ref [18]. The technical specifications of a 4-DOF exoskeleton robot are detailed in Table 1. All the robot links are made of aluminum, so it is lightweight and very durable. In Fig. 1(b), the counterweight is attached to the joint equivalent joint 1 of the shoulder joint as shown in Fig. 1(a). This counterweight is for compensating the gravity load, so the actuator size can be reduced significantly.

Table 1. Technical data of the 4-DOF exoskeleton robot.

\begin{tabular}{lllll}
\hline & Joint 1 & Joint 2 & Joint 3 & Joint 4 \\
\hline Range of Motion (ROM) & 100 & 120 & 140 & 115 \\
Motor type & Brushless motor & Brushless motor & Brush motor & Brushless motor \\
Motor torque continuous & $0.533 \mathrm{Nm}$ & $0.533 \mathrm{Nm}$ & $0.106 \mathrm{Nm}$ & $0.533 \mathrm{Nm}$ \\
Transmission ratio & 12.5 & 12.5 & 19 & 8.75 \\
Load torque continuous & $6.66 \mathrm{Nm}$ & $6.66 \mathrm{Nm}$ & $2.01 \mathrm{Nm}$ & $4.66 \mathrm{Nm}$ \\
Robot Link mass & $1.407 \mathrm{~kg}$ & $0.608 \mathrm{~kg}$ & $0.732 \mathrm{~kg}$ & $1.123 \mathrm{~kg}$ \\
Total mass (Moving part) & $3.4 \mathrm{~kg}$ & & & \\
\hline
\end{tabular}

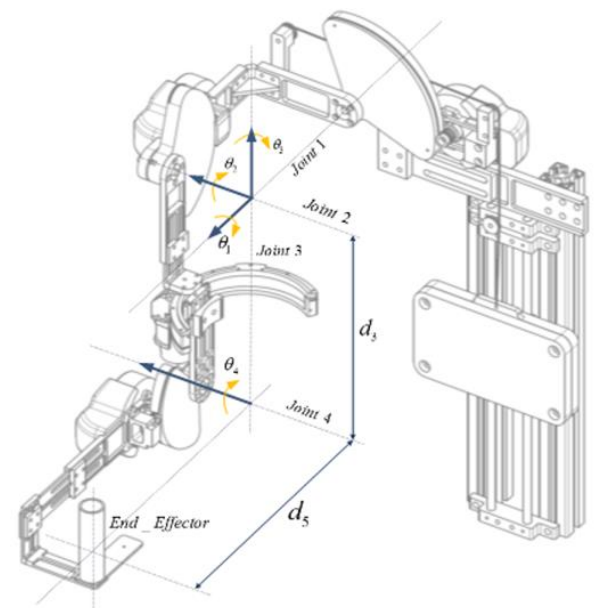

(a)

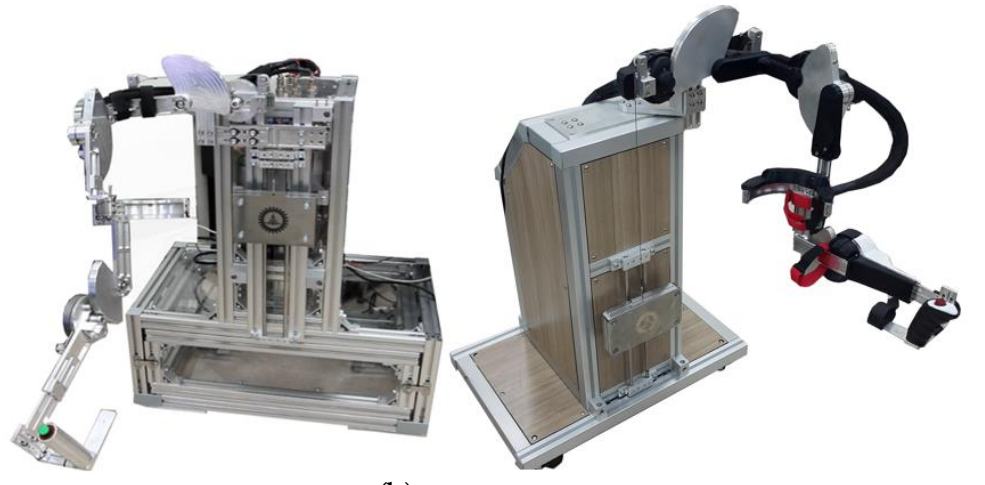

(b)

Fig. 1. (a) The kinematic parameters and the assignment of coordinate frames for the 4-DOF upper limb exoskeleton robot. (b) The 4-DOF upper limb exoskeleton robot, CUREs, for stroke rehabilitation used in this study.

\section{Control Strategy of Each Joint}

Typical dynamic model of serial type manipulator arms is very non-linear. Due to the nature of operation, typical speed for stroke rehabilitation is not that high, the non-linear terms as Centripetal and Coriolis can be neglected. This can simplify the dynamic model as a linear model. And each joint can be controlled independently. The control strategy in this study is the active assistive strategy. The controller is implemented on each joint of our CUREs robot. This controller is active assistive control strategy. Both assist-resist and resistive mode are implement. The assist-resist mode of operative is suitable for a patient who has severe condition or with difficulty moving his hand or physically weak person. The robot will help the patient by 
guiding his hand along a pre-defined path even though he could not do it by himself. The robot will either assist or resist while the patient performs the training. With this control strategy, the patient will gain, indirectly, mentally more confident after going through training sessions.

The power transmission mechanism between load (links plus joint) and actuator is cable and pulley. The cable has some small compliance. One of the major role of this compliance is act as a safety component in the control scheme. It can absorb some vibration energy and can reduce impact load. So, dynamic stability of the driving system can be improved especially for impact load. More details concerning about the safety can be consulted in [24]. Figure 2 is the block diagram of our control strategy. It is an impedance control cascaded with torque control. The similar controller was implemented in the lower extremity powered exoskeleton (LOPES) for gait rehabilitation [14].

The inner loop is a velocity control loop. It is the PI-controller closed with a filtered differentiation of the encoder signal in contrast with using Hall-based velocity sensor as discussed in [24]. Our approach is easy to implement but will induce some time delay in the velocity control loop about half of the sampling time. From our experiment, the effect of this time delay has not shown any significant problem within our design bandwidth. The outer loop is the force control loop. It also used the PI-controller closed with load torque feedback $T_{L}$. The load torque in the feedback loop is obtained by measuring the input current of the power supply or motor driver of the brushless DC servomotor multiply by torque constant $K_{a}$ of the motor.

For impedance control, the desired torque $T_{L, d}$ can be generated by a simple proportional controller which is the function of the constant stiffness $P$ and angular error between the desired angle $\theta_{d}$ and the actual angle $\theta_{L}$.

$$
T_{L, d}=P\left(\theta_{d}-\theta_{L}\right)
$$

The desired torque in Eq. (1) is a linear function. This control acts like a spring, the output force increase constantly if the angular position error increase. However, we need the robot to help or guide a movement of the patients arm with the minimal force (Assistance-as-Needed, $\mathrm{AaN}$ ). In case of a small angular position error, the assistance torque is adjusted by using the virtual wall control strategy based on the concept studied in [25] and [26] as following:

$$
T_{L, d}=\operatorname{sgn}\left|\theta_{d}-\theta_{L}\right| K_{p} e^{\left|\theta_{d}-\theta_{L}\right| K_{f}}-1
$$

where $K_{p}$ and $K_{f}$ are the gains of the impedance part that can be adjusted to obtain a suitable desired torque of the outer loop control, which is the torque control loop, as shown in Fig. 2. The desired torque is adjusted to suit for any training session. The torque $T_{L}$ is the torque appears at the load side of the exoskeleton arm. This torque can be measured by measuring the current of the motor driver. Normally, the value of gain $K_{p}$ should be set equal to the motor torque constant and much smaller than the gain $K_{f}$.

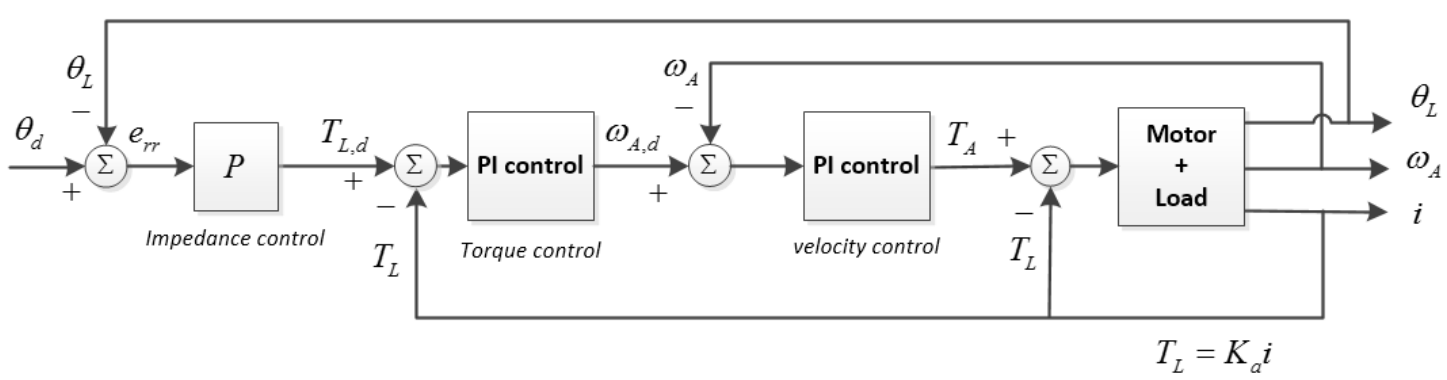

Fig. 2. Block diagram of the Assistive-resistive control strategy. 
In Fig. 3, the desired torque from Eq. (1) (blue line) and the desired torque from Eq. (2) (red line) are presented. It can be shown that the desired torque $T_{L, d}$ in Eq. (2) is lower than Eq. (1) if error is smaller than 0.125. And the desired torque $T_{L, d}$ in Eq. (2) is higher than Eq. (1) if error is higher than 0.125. This intersection point can be adjusted by adjusted gains $K_{p}$ and $K_{f}$. This desired torque will be used as reference torque command in the torque control loop.

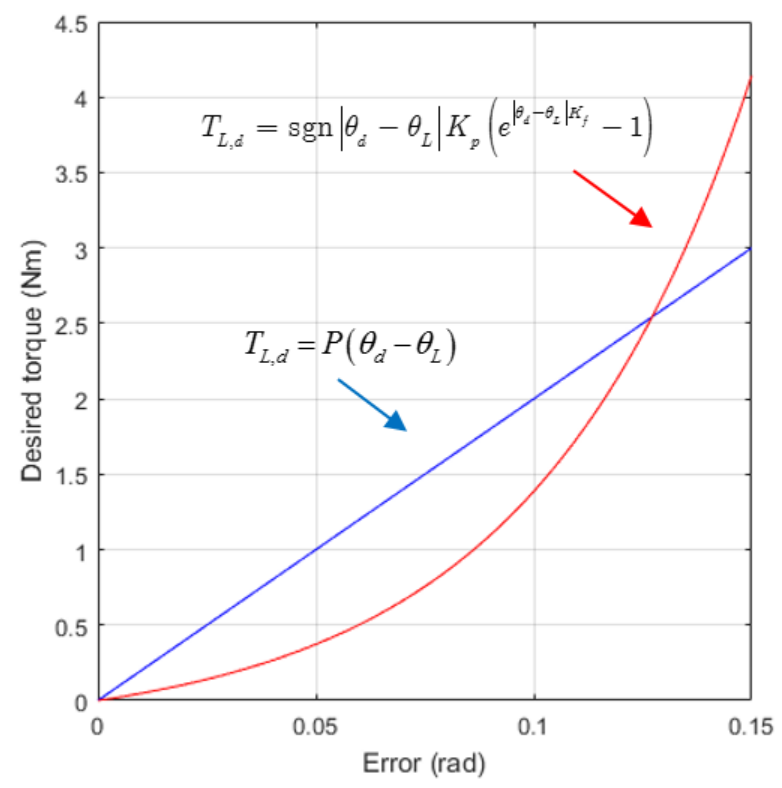

Fig 3. The desired torque, from impedance models, used as reference torque command in the torque control loop.

The advantage of this control strategy, as long as the joint torques do not exceed the torque limit, the motion of the robot arm can follow the desired trajectory. In case of the joint torque exceed the torque limit of each joints, the motion of the robot will be effected by these external forces (exerted by a patient). The robot joint torque will be controlled so that the control torque will not exceed the maximum torque set by the torque limit. This controller make the robot more safety when compare to the rehabilitation robot which using only motion control strategy [13]. Moreover, the measurement of load torque $T_{L}$, used in our control strategy, is measured by using the current measurements of the motor drivers. This will simplify the torque measurement compare to the typical torque measurement, using expensive force sensors, in most of the rehabilitation robots. For example, the gait rehabilitation robot [14], which also need force measurement in its control loop, using the direct force sensors.

\section{Experimental Setup}

The patient using CUREs robot will perform training in front of a large graphic display as shown in Fig. 4. Figure 4 shows a fix-based model of rehabilitation robot while Fig. 1 is a movable system. Both the fix-based model and movable model have the same configuration arm. The arm of the CUREs robot is fixed on an aluminum frame behind the patient. The shoulder joint consists of three revolution joints where the axis of rotation of the revolute joints intersect at the same point. The intersection of the first three joints of the robot must be coincident with the patient shoulder joint. The rotation axis of the fourth joint of the robot (elbow joint) must be aligned to the elbow joint of the patient. The patient hand will hold the handle which is attached to the end of the exoskeleton arm. The upper arm (between shoulder and elbow) and the lower arm (between elbow and wrist) of the patient are tied with the robot arm using Velcro tapes so that the patient arm will align with the exoskeleton arm.

In this paper, a 4DOF upper limb exoskeleton robot has been tested with a normal healthy person and 5 stroke patients with Brunnstrom level 1 and level 2. The 3 patients have right hemiparesis and the other 2 
patients have left hemiparesis [19]. The main purpose of this experiment is mainly to follow the clinical trial procedure level 3. To qualify as a commercial product, we need to demonstrate that there should not have any negative side effect for the patients who use the rehabilitation robot. We used the Fugl-Meyer Assessment Upper Extremity Scale (FMA-UE) for upper extremity recovery assesment [27] and the Thai version Motor Assessment Scale (MAS) for upper extremity function assessment [28]. The results are summarized in section 6.

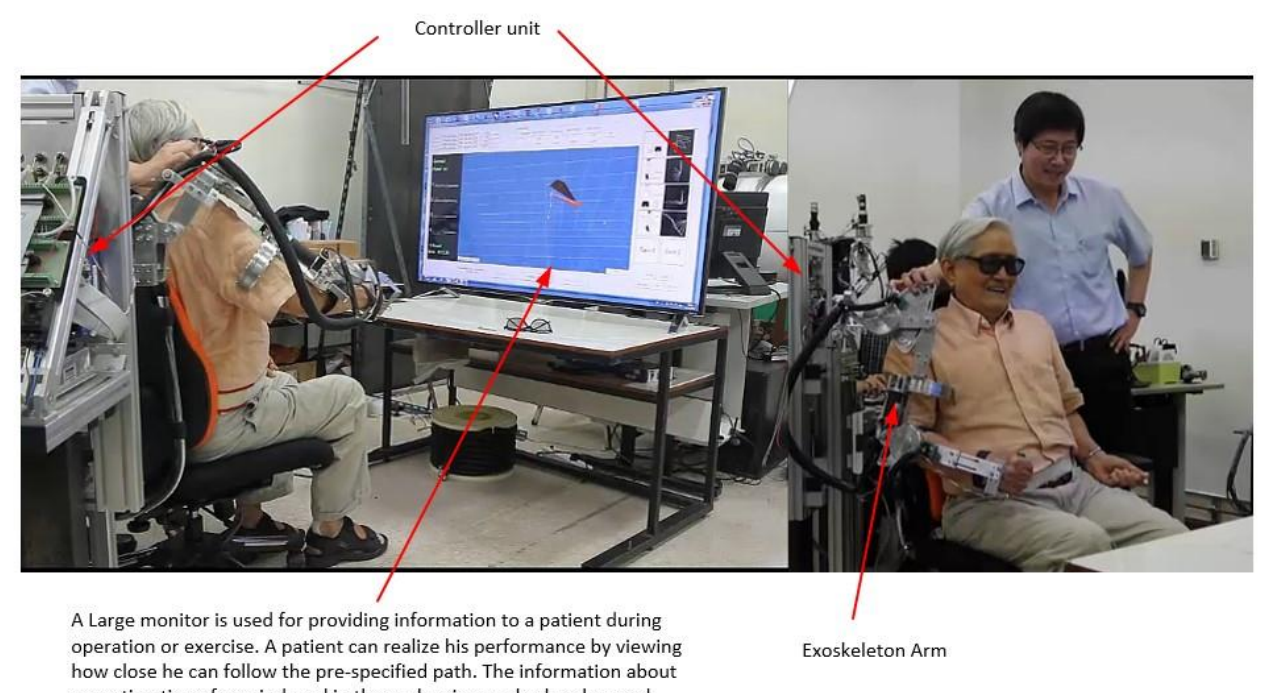

Fig. 4. The CUREs (Chulalongkorn University Rehabilitation Exoskeleton system). This is a fixed-base model.

For assistive control strategy, the desired trajectory or rehabilitated training pattern for each specific patient need to be assigned first by a medical doctor. The therapist can generate the desired trajectory by guiding the patient arm, which tied with exoskeleton arm, according to the assigned pattern. While therapist guiding the patient with robot arm, points along the guild path can be captured and stored. These via points are shown on the operation monitor as shown in Fig. 5 and the trajectory can be generated. This trajectory is stored and can be reused anytime. Figure 5(a) shows the recorded points during therapist-guided and the training trajectory will be generated. The pink line in Fig. 5(b) and Fig. 5(c) is represented the reference arm's posture of the exoskeleton that the patient need to follow as the desired training trajectory. While the patient is wearing the exoskeleton arm robot and try to move his arm along the desired trajectory, he can observe his arm posture compared to the reference posture in real time as shown in Fig. 5. Moreover, the graphs of the operation torque at each joints of robot arm are measured and display in real-time on the same display monitor. If the patient cannot move his arm along the assigned trajectory, the exoskeleton will assist by applying more torque to help the patient to move his hand. In opposite, if the patient has ability to move his hand along the assigned trajectory, the robot will not generate any more torque to help the patient. The information of the training activity is graphically shown in real-time on the display monitor and also automatic saved as a file which can be reviewed by the medical doctor to follow the performance of the patient.

The typical training procedure is shown in Fig. 6. The desired trajectory for the patient is preprogrammed as mention previously. The desired trajectory and arm's postures are display as shown in Fig 5 . This particular training is mainly for elbow joint while the other three joints at the shoulder have a very small movement as shown in Fig. 6, the series of pictures from (a) to (d) show the patient is performing the elbow training. So, the patients will focus only their elbow movement. This training is part of the clinical trial level 3 of the medical ethic to demonstrate no sign of side effect to the patient. The patient participated in $30 \mathrm{~min}$ of robot training and $30 \mathrm{~min}$ of conventional upper-limb training. The significant parameter such as the desired position $\theta_{d}$, actual position of the robot joints $\theta_{L}$, time $t$ and load torque at each motor $T_{L}$ are recorded. More detail about clinical trial will be explained in section 6 . 

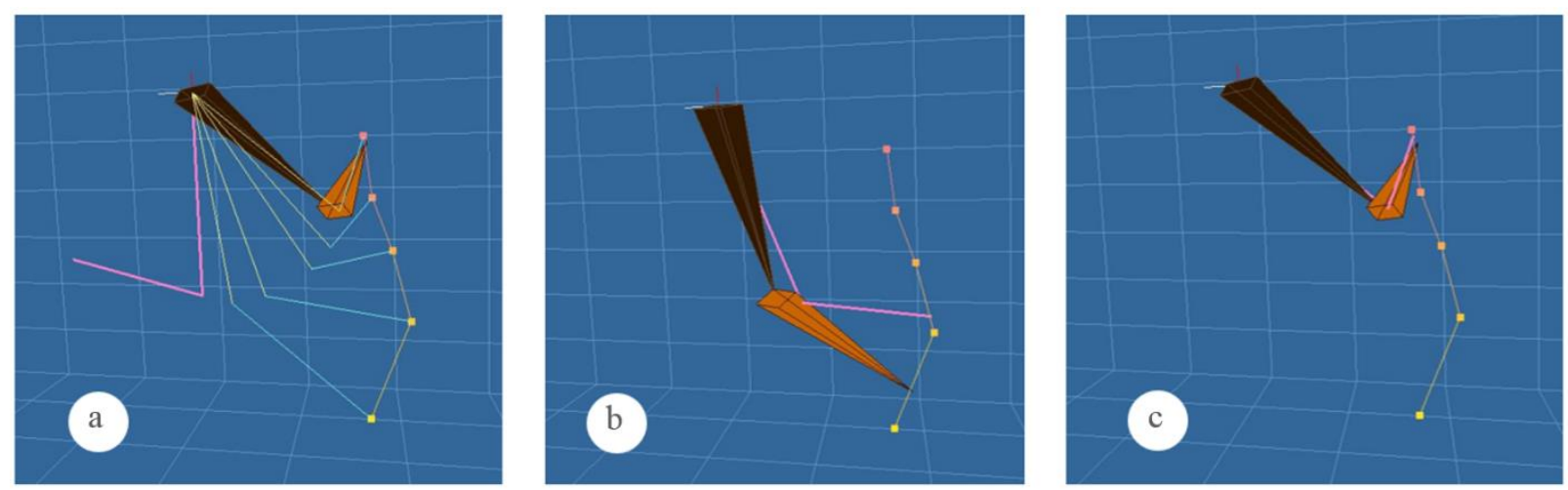

Fig 5. (a) shows a training trajectory generated from record points. (b) shows the deviation of the actual robot arm motion (solid link) from the desired trajectory. (c) shows almost perfect tracking between patient (robot arm) and desired trajectory.

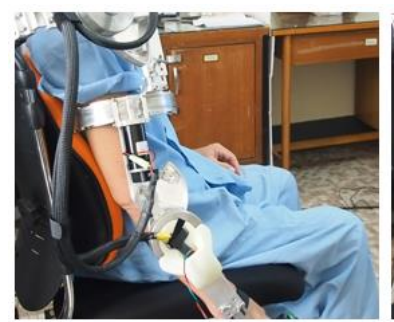

(a)

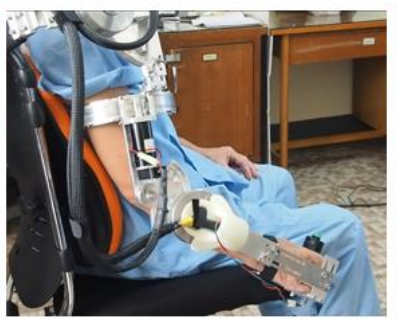

(b)

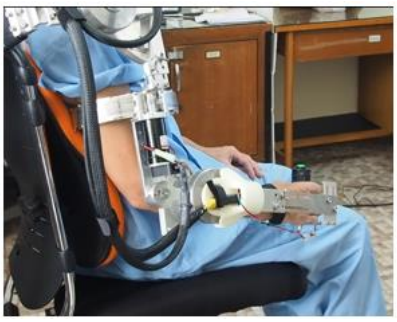

(c)

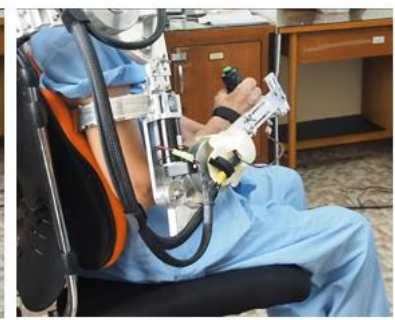

(d)

Fig. 6. Series of pictures demonstrate elbow training of a stroke patient, who is right hemiparesis, with our 4-DOF exoskeleton robot.

\section{Result}

In this experiment, time $t$, desired position of all joints $\theta_{d}$, the actual position of all joints $\theta_{L}$, and load torque at the motors $T_{L}$ are recorded every period of 100 milliseconds. The desired angular position and the actual angular position of each joint are presented in Fig. 7, for normal or healthy person, and Fig. 8, for a stroke patient. The plots are arranged in row format starting from joint 1 to joint 4 . The blue line is reference angular position and the red line is the actual angular position. As shown in the plots, both values are very close together, this is demonstrate the performance of the controller. Figures 7 and 8 show that there are little movement in the first joint (abduction/adduction) and the second joint (flexion/extension) but more movement in the third joint (internal/external rotation) and the most movement is in the fourth joint (elbow flexion/extension). Moreover, it shows that the 4-DOF exoskeleton arm can perform very well to help both healthy subject and stroke patient to follow the pre-defined trajectory. 

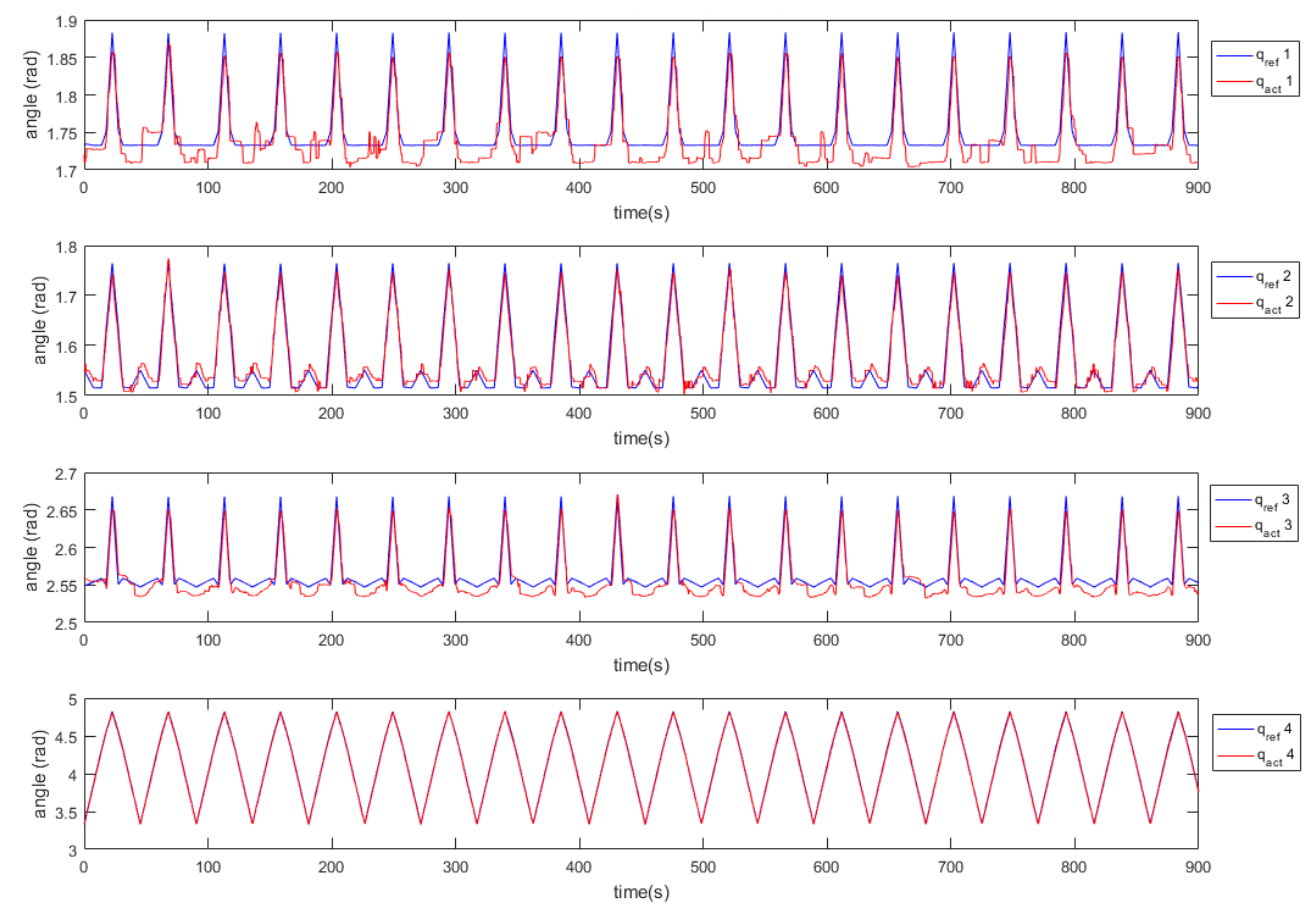

Fig. 7. Time history of desired angular position and actual angular position of the healthy subject.
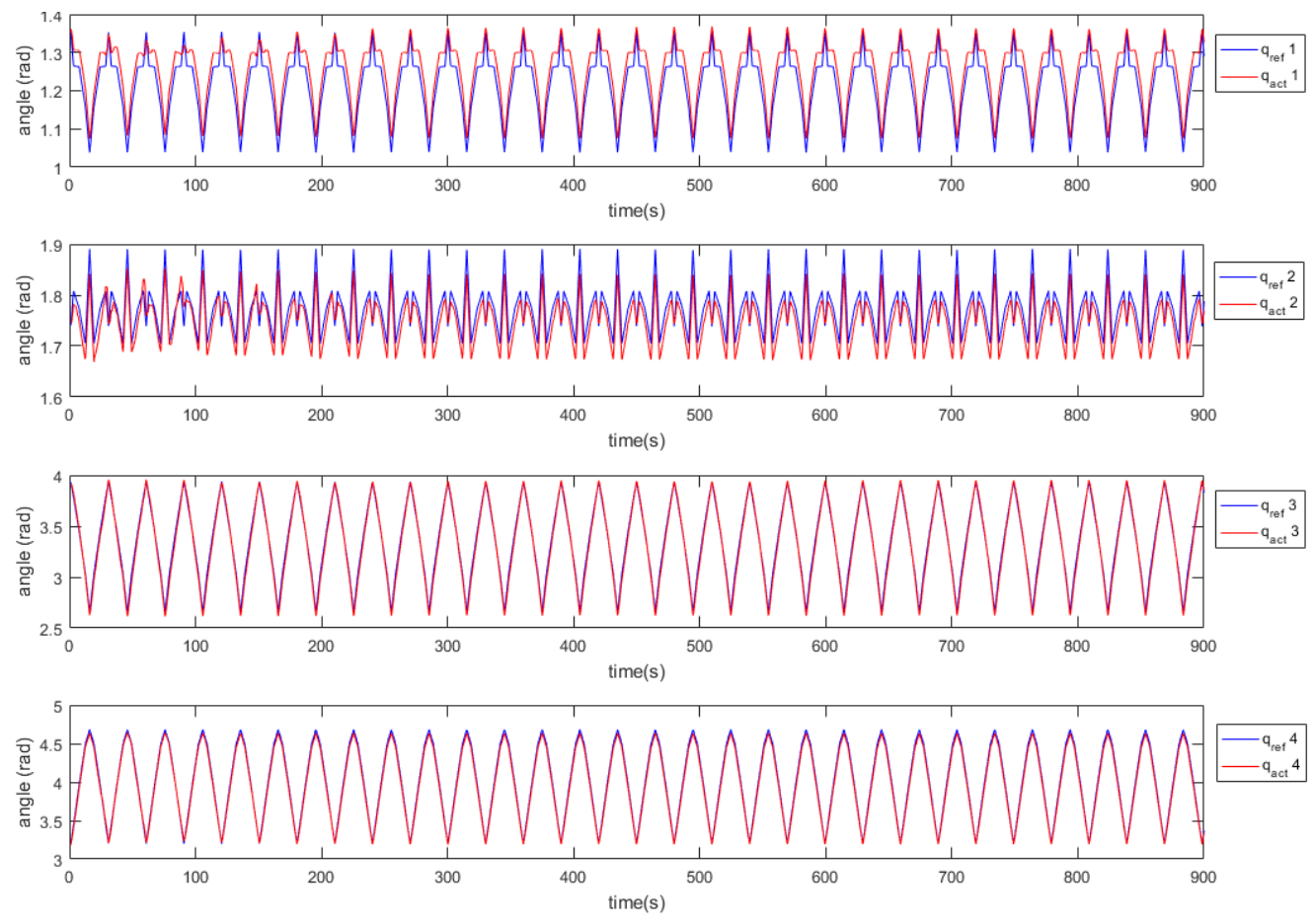

Fig. 8. Time history of desired angular position and actual angular position of the stroke patient.

Figures 9 and 10, the measurement of load torque of healthy subject and stroke patient at each motors are presented respectively. The graphs are arranged in rows format by starting from load torque at joint 1 (top row) to joint 4 (last row). The result from Fig. 9 showed that measurement of load torques had small magnitude. It means that the healthy subject can move this arm by little helped of the robot. 

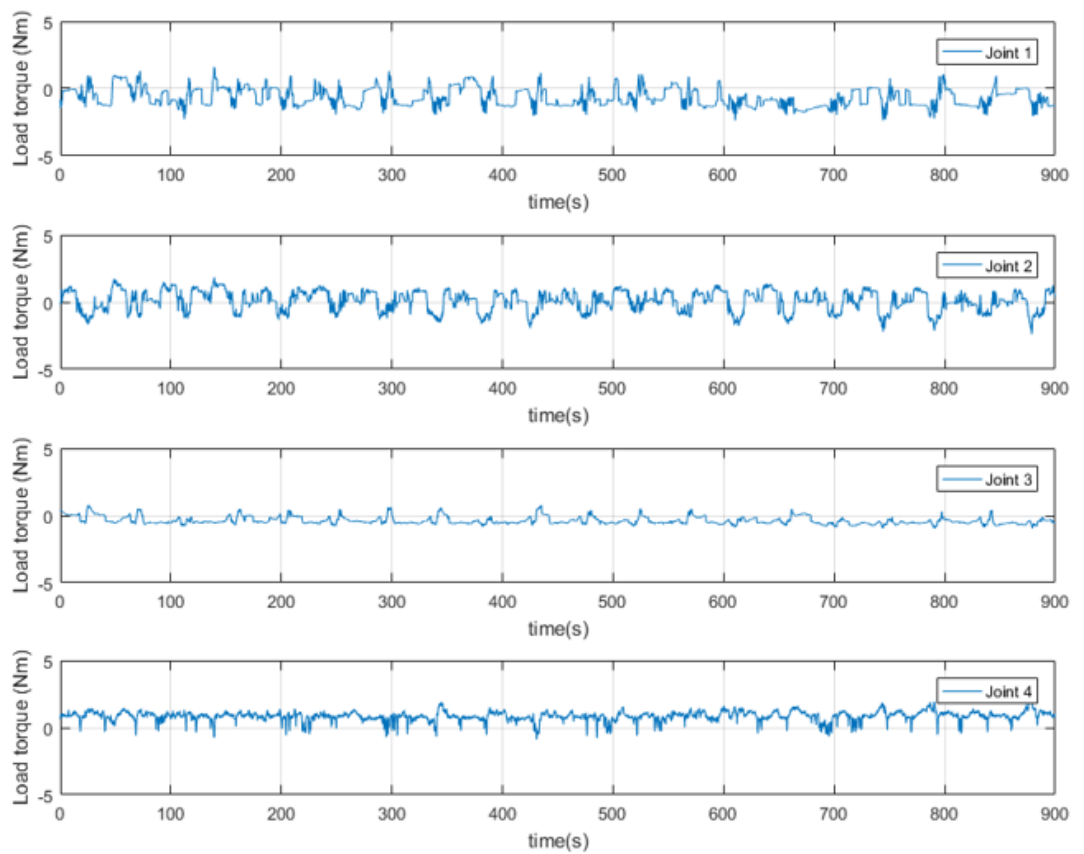

Fig. 9. Time history of the motor torque and the angular position error of the healthy subject.

Figure 10 shows the time history of the motor torque and the angular position error of the stroke patient. From the plot, we notice that the load torques have a repetitive pattern. During the first 100 seconds, the load torques at joint 4 (elbow) are very low. This shown that the stroke patient tried to move his arm by himself and very small help from the exoskeleton arm. After that, he let the exoskeleton arm to assist his arm motion to follow the desired trajectory.
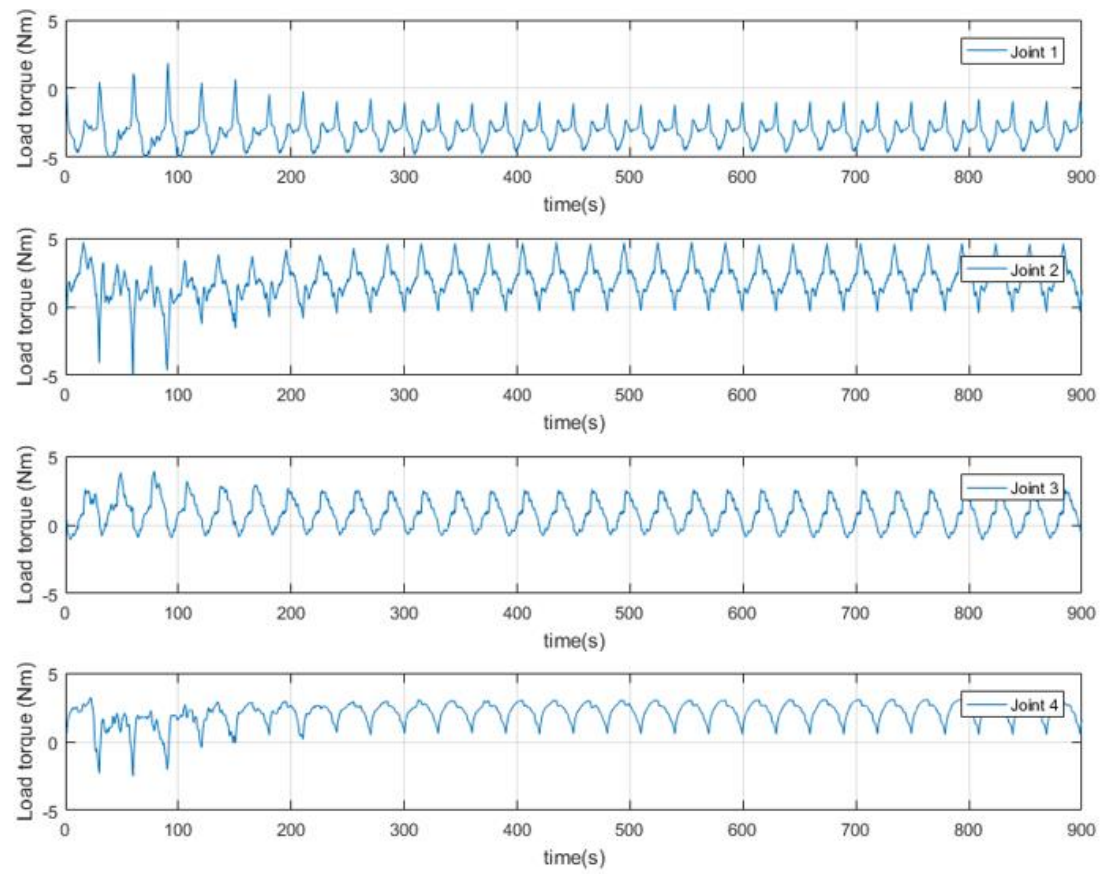

Fig. 10. Time history of the motor torque and the angular position error of the stroke patient.

Figures 11 and 12 show histograms of the load torque distribution. This histogram is very useful for summarizing a large amount of data. Figure 11 covers three days of training of the healthy subject. The green line is the median of the load torque distribution. The higher value of the median means that the exoskeleton 
assists more of the movement of patient arm. On the other hand, the lower value of the median means the patient try to move his arm by himself to follow or track the desired trajectory or the exoskeleton assists less. Figure 12 shows the histograms of the load torque distribution of elbow joint of the first patient during the first 7 days of training. Because of the large movement is mainly in the 4th joint (elbow joint), the load torque at the 4th joint is the best option to see the rehabilitation progress as shown in Fig. 12. These results show some improvement after the short period of training.
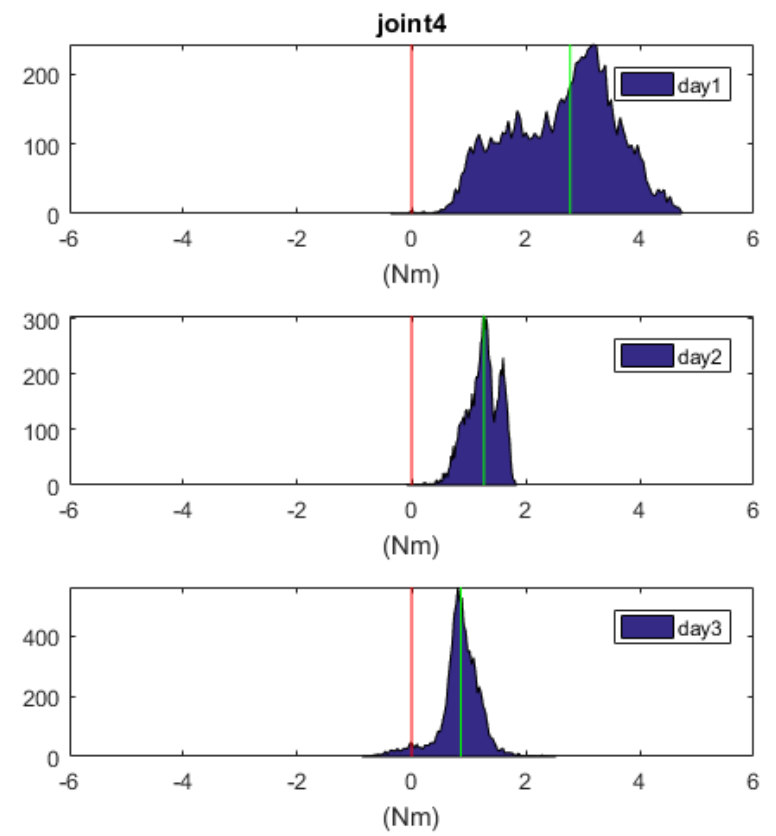

Fig. 11. The histograms of the load torque distribution of the 4th joint of the healthy subject in 3 days.

Instead of histogram, we can also show the load torque of the training in candlestick charts as shown in Figs. 13 - 18. Figure 13 is the median of load torque of the healthy subject during 3 days. The figure has four columns, each column represents each joint torque of the exoskeleton arm. A candlestick shows a maximum value, a minimum value and a median value of load torque every day of training. Due to the lower torque of the exoskeleton, this is the sign of some progress of the patient capable of controlling his arm by himself to track the desired trajectory.

Similarly to Fig. 13, Figs. 14 - 18 present the candlestick chart of load torque of the $1^{\text {st }}$ patient in 7 days, $2^{\text {nd }}$ patient in 6 days, $3^{\text {rd }}$ patient in 9 days, $4^{\text {th }}$ patient in 4 days, and $5^{\text {th }}$ patient in 4 days, respectively. Every patients, except $3^{\text {rd }}$ patient, shown some improvement after training within a short period of time. 

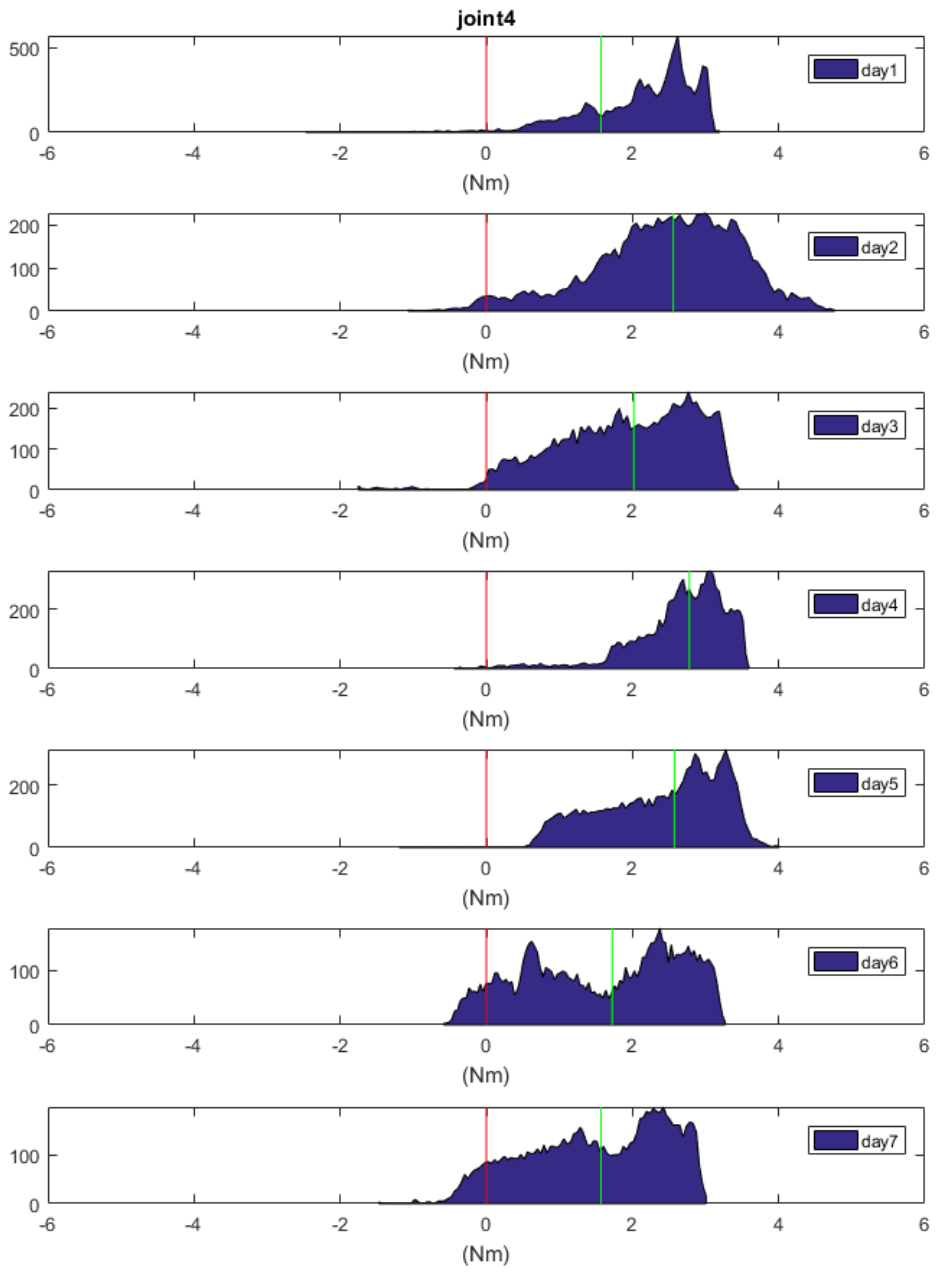

Fig. 12. The histograms of the load torque distribution of elbow joint of the first patient during 7 days.
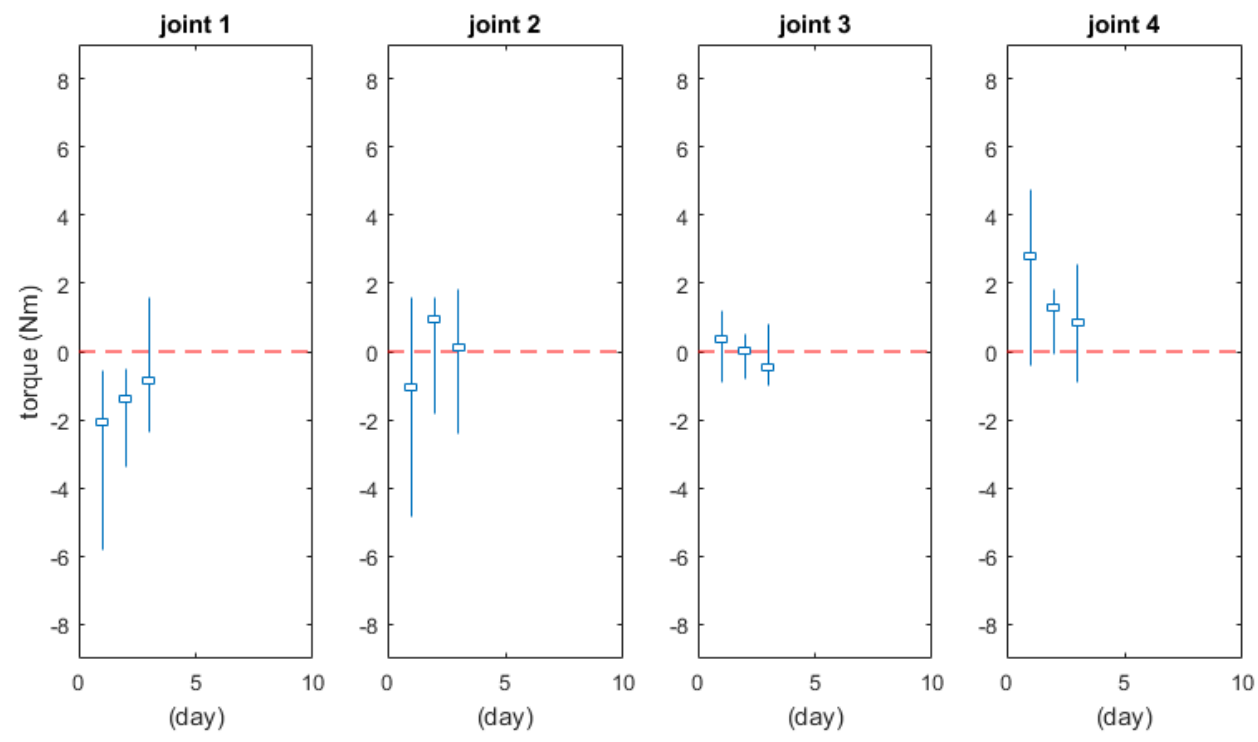

Fig. 13. The median of load torque of the healthy subject for 3 days. 

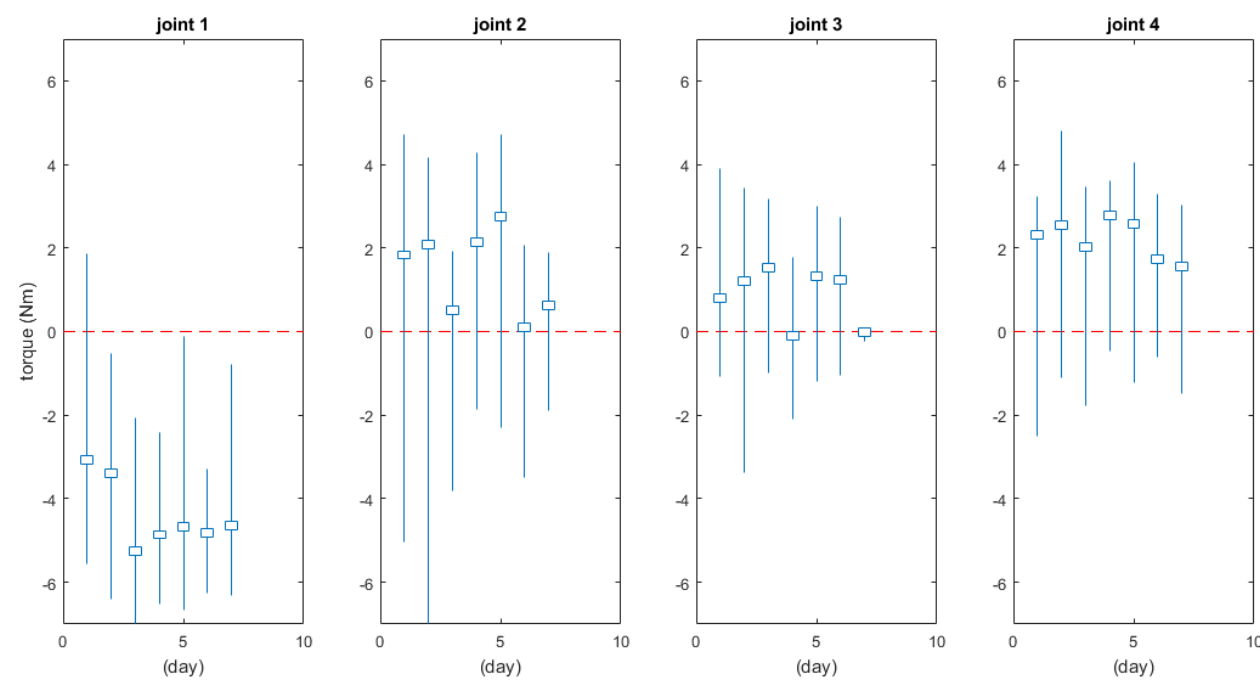

Fig. 14. The median of load torque of the 1 st patient for 7 days.
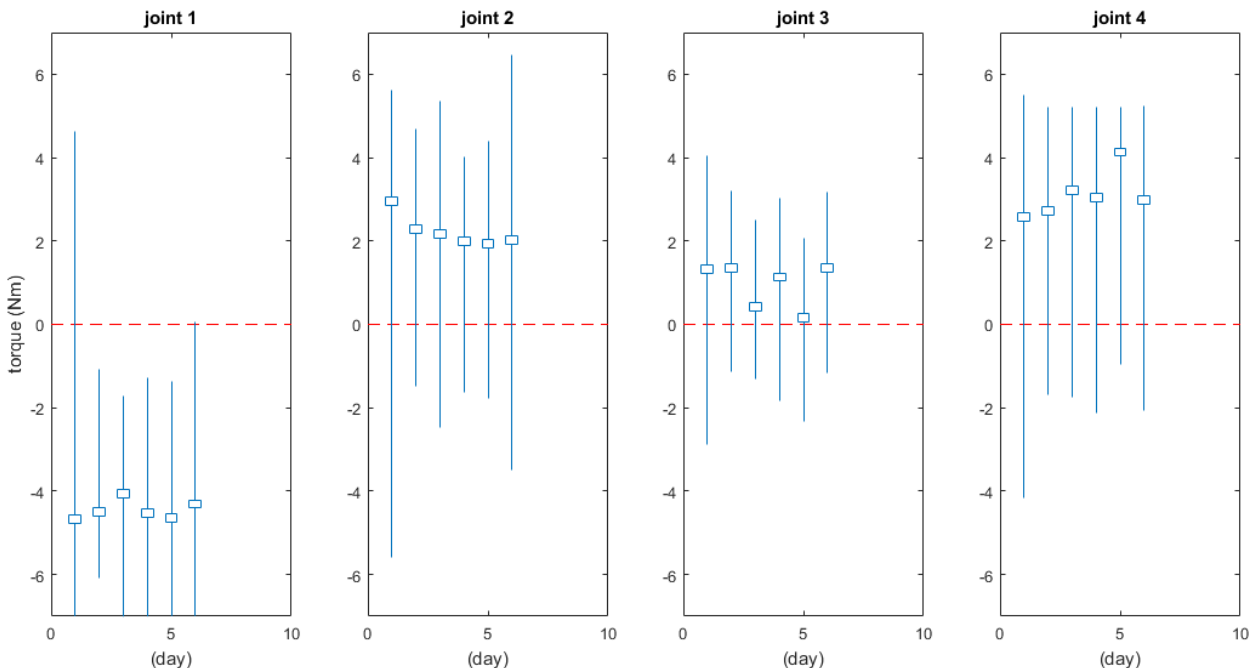

Fig. 15. The median of load torque of the 2 nd patient for 6 days.
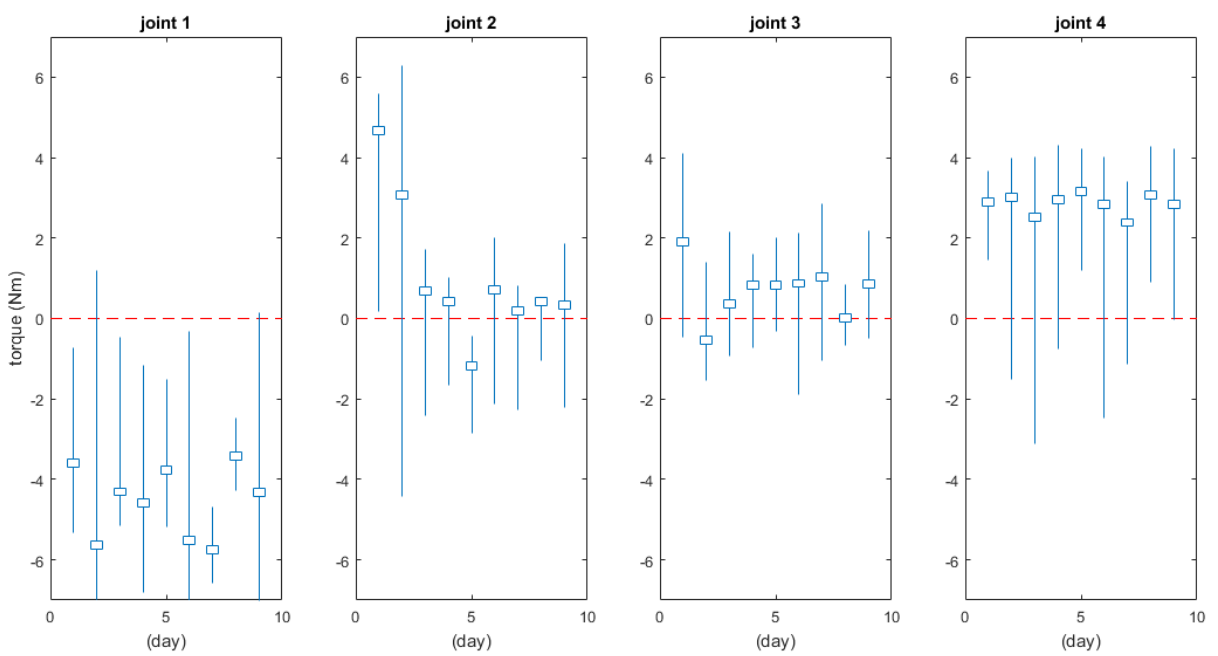

Fig. 16. The median of load torque of the 3rd patient for 9 days. 

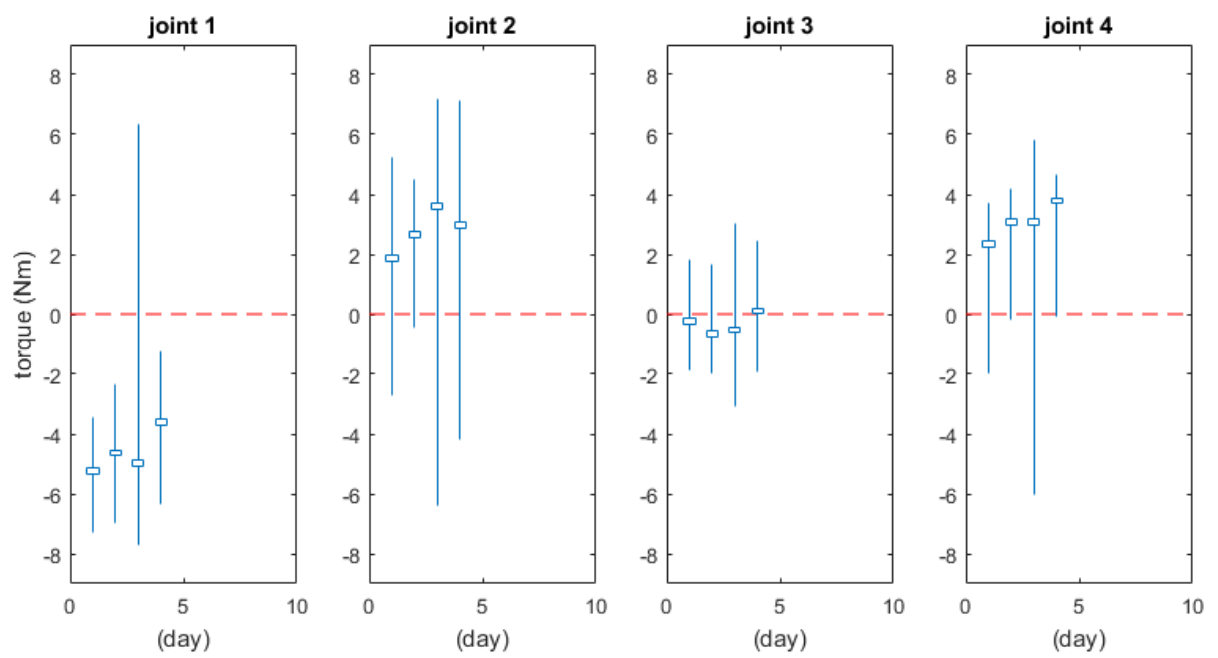

Fig. 17. The median of load torque of the 4 th patient for 4 days.
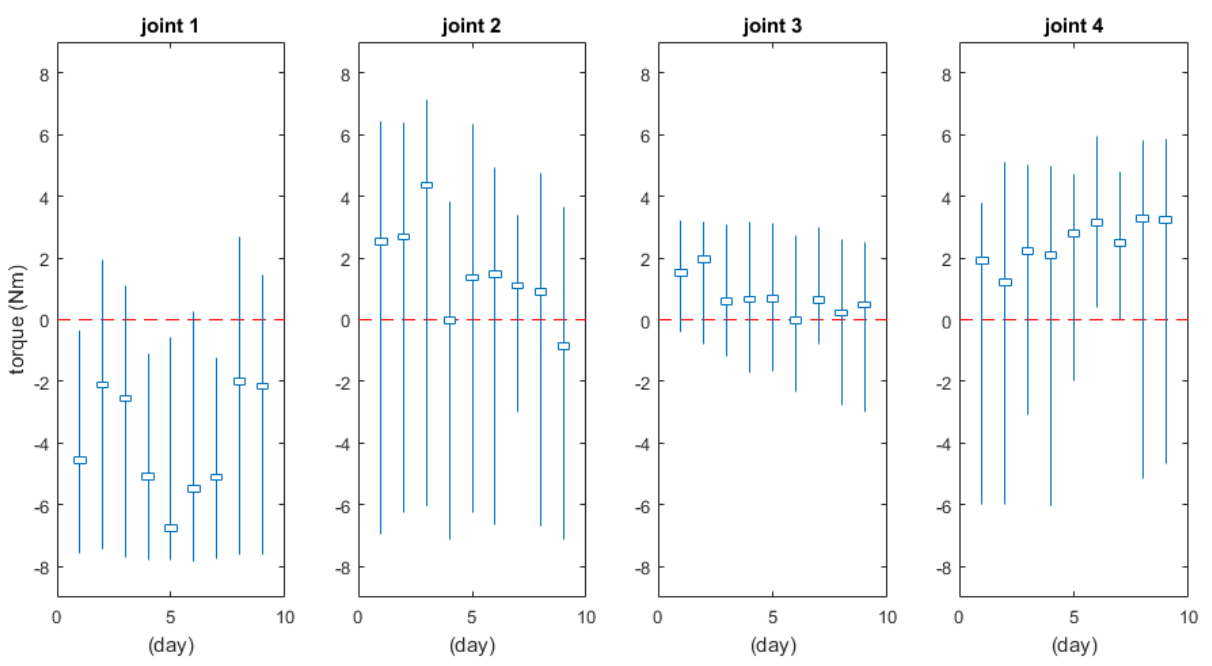

Fig. 18. The median of load torque of the 5th patient for 9 days.

\section{Verification of Training with the Exoskeleton Robot}

This project is verified by a team of medical doctors and the author under a pilot project named "Upper Extremity training with CUREs Robot in Subacute Stroke: A Pilot Study" [19]. The main purpose of this clinical trial is to follow the medical ethic and must be approved by institutional ethical committee to assure that there will be no sign of negative side effect for the patients who use this rehabilitation robot. This clinical trial is performed by a group of medical doctors and therapist. The medical doctors will assign the training activities based on patient condition. The therapist is responsible for the use of the rehabilitation robot and setting up the reference trajectory based on the suggestion of the rehabilitation doctor. The result of clinical trial of the project is summarized in this paper for the completeness of the development of the exoskeleton arm.

As mention in previous section, five subacute stroke patients participated in a pilot study. They were admitted for intensive rehabilitation at the Thai Red Cross Rehabilitation Center. Inclusion criteria were 1) $1^{\text {st }}$ ever hemiplegia/hemiparesis stroke 2) onset from 1 to 6 months 3) Brunnstrom stage of upper extremity stage I to V and 4) sit with/without support for a least 30 minutes. Patients were excluded if they have 1) communication problem due to aphasia or severe dysarthria 2) severe fixed contracture of shoulder and elbow 3) nociceptive or neuropathic pain in affected upper extremity 4) modified Ashworth scale 3 or 45 ) severe perception deficit 6) cognitive impairment measuring with Thai Mental state Examination and 7) moderate to severe depression measuring with Thai Geriatric Depression Scale. They were informed consent 
and signed the form before participating in our study. This study was approved by institutional ethical committee.

We used the Fugl-Meyer Assessment Upper Extremity Scale (FMA-UE) for upper extremity recovery assesment [27] and the Thai version Motor Assessment Scale (MAS) for upper extremity function assessment [28]. We assessed all patients before participation and after 2 weeks training. Total score of FMA-UE motor function (upper extremity, wrist, hand, coordiantion/speed) is 66. Total score of FMA-UE sensation, passive joint motion and joints pain are 12, 24 and 24, consecutively. Intensive rehabilitation program included physical therapy, occupational therapy, speech therapy, psychotherapy and orthotic service. Regarding upper extremity rhabilitation, they particiapated in $30 \mathrm{~min}$ of conventional upper extremity training and $30 \mathrm{~min}$ of robotic training, 5 days per week for 2 consecutive weeks. The robot automatically generated assistive force depending on the muscle power of each patient.

Five subacute stroke patients completed the robotic training program. Onset was from 1 to 3 months after stoke. Four patients had Brunnstrom stage I at baseline. One patient had stage II. Baseline characteristic of them was shown in Table 2, FMA-UE total score was improved in all patients after 2 weeks of conventional upper extremity training with robotic training. FMA-UE motor score at baseline (1) and 2 weeks after training (2) was shown in Table 3. However, MAS was not changed at 2 weeks after training in all patients. No major complication was found, except temporary muscle soreness. The Fugl-Meyer Assessment Upper Extremity (FMA-UE) sensation, joint motion and pain score are shown in Table 4.

This study means that the robot is safe to use in the rehabilitation activities. In most countries, this study is enough for commercializing the robot systems. Next level or the level 4 of the medical ethic is to study about the curing performance of the robot. There are about 60 volunteer patients in the study. To cover all the rehabilitation activities, the study will last more than a year. And it is being done by a group of medical doctors and therapist. The results of the level 4 study will be published in a Medical Journal.

Table 2. Baseline characteristics of participant.

\begin{tabular}{ccccccc}
\hline No & Age (year) & Gender & Onset (day) & Weak side & Stroke type & Lesion \\
\hline $\mathbf{1}$ & 57 & Female & 90 & Right & Hemorrhage & Thalamus \\
$\mathbf{2}$ & 47 & Male & 60 & Right & Hemorrhage & Thalamus \\
$\mathbf{3}$ & 51 & Male & 45 & Right & Hemorrhage & Basal ganglia \\
$\mathbf{4}$ & 50 & Male & 67 & Left & Hemorrhage & Basal ganglia \\
$\mathbf{5}$ & 55 & Female & 32 & Left & Infarction & Pons \\
\hline
\end{tabular}

Table 3. The Fugl-Meyer Assessment Upper Extremity (FMA-UE) Motor score.

\begin{tabular}{lccccccccccc}
\hline No & \multicolumn{3}{c}{ Total score } & \multicolumn{2}{c}{$\begin{array}{c}\text { Upper } \\
\text { extremity }\end{array}$} & Wrist & Hand & \multicolumn{2}{c}{$\begin{array}{c}\text { Coordination } \\
\text { /speed }\end{array}$} \\
\cline { 2 - 13 } & \multicolumn{1}{c}{} & $\mathbf{2}$ & $\boldsymbol{\Delta}$ & $\mathbf{1}$ & $\mathbf{2}$ & $\mathbf{1}$ & $\mathbf{2}$ & $\mathbf{1}$ & $\mathbf{2}$ & $\mathbf{1}$ & $\mathbf{2}$ \\
$\mathbf{n}$ & 59 & 72 & 13 & 2 & 7 & 0 & 0 & 5 & 10 & 3 & 3 \\
$\mathbf{2}$ & 52 & 60 & 8 & 2 & 8 & 0 & 0 & 4 & 6 & 4 & 4 \\
$\mathbf{3}$ & 50 & 52 & 2 & 0 & 0 & 0 & 0 & 0 & 0 & 4 & 4 \\
$\mathbf{4}$ & 52 & 58 & 6 & 2 & 3 & 0 & 0 & 0 & 4 & 4 & 4 \\
$\mathbf{5}$ & 67 & 81 & 14 & 5 & 18 & 0 & 0 & 0 & 1 & 4 & 4 \\
\hline
\end{tabular}

Table 4. The Fugl-Meyer Assessment Upper Extremity (FMA-UE) Sensation, joint motion and pain score.

\begin{tabular}{ccccccc}
\hline No & \multicolumn{2}{c}{ Sensation } & \multicolumn{2}{c}{ Passive joint motion } & \multicolumn{2}{c}{ Joint pain } \\
\cline { 2 - 7 } & $\mathbf{1}$ & $\mathbf{2}$ & $\mathbf{1}$ & $\mathbf{2}$ & $\mathbf{1}$ & $\mathbf{2}$ \\
$\mathbf{n y y y y y y}$ & 6 & 8 & 24 & 24 & 19 & 20 \\
$\mathbf{2}$ & 6 & 6 & 20 & 20 & 16 & 16 \\
$\mathbf{3}$ & 6 & 6 & 20 & 22 & 20 & 20 \\
$\mathbf{4}$ & 6 & 7 & 20 & 20 & 20 & 20 \\
$\mathbf{5}$ & 10 & 10 & 24 & 24 & 24 & 24 \\
\hline
\end{tabular}




\section{Discussion}

We developed a 4-DOF exoskeleton robot, CUREs, for stroke rehabilitation. The active assistive control strategy with pre-define trajectories is used as the main dynamic controller. The parameters, such as the desired trajectory, the actual trajectory and the operation torque, can be monitored in real-time. The result showed that the robot can assist the patient's arm following along the desired trajectory as showed in figure 8. The desired trajectory can be generated by connection two consecutive points with a straight line with constant velocity. So, the arm movement might not be that smooth at the connecting points due to discontinuity of the via point. The discontinuity occurs because of the change of direction. However, we could improve the desired trajectory by using the minimum jerk movement profile as explained in [29].

Due to the reference trajectory was fixed, the patient need to move his arm to follow the trajectory. With the proposed impedance control, within some vicinity away from the reference trajectory, there are automatic switch between the perform of the robot and the perform of the patient based on the variable deviation from the reference trajectory. Due to the volunteer patients have severe condition, they cannot move their upperlimb. With this control strategy, results shown in the clinical trial, he can gain ability to recover some muscle strength within the period of experiment without any negative side effect. Therefore, this active assistive control strategy is probably one of the suitable choice in this class of rehabilitation.

The result from Figs. $9-10$ showed the load torques over time. From this result, the therapist could evaluate performance and intention of a patient. If the patient try to move by himself, the load torques might be around zero. But if the patient does not try to move (free fall his/her arm), the load torque will increase. From row 4 in Fig. 10 (elbow joint), load torque had gone to zero about 100 seconds. And then, load torque at joint 4 had had a repetitive pattern till the end of the test. This result seem that the patient had the attempt to move his arm about 100 seconds and then the patient probably allowed the robot guiding his arm along the desired trajectory. Although the patient did not try to move by himself, over the assistance of the robot, the muscles and connective tissue could be stretched. This is thought to be essential for provoking motor plasticity [30], and stretching can help prevent stiffening of soft tissue and reduce spasticity, at least temporarily [31].

Figures 11-12 are the histograms of the distribution and median of load torque recorded every day, the therapist can investigate the performance or progress of individual patient. And he can stimulate patients to be more serious about the training if the progress is not improved. The result from Figs. 14-18 showed that the 1st patient and 3rd patient seem to have more progress. This improvement of the motor recovery of the patients might be that the patients had been motivated by therapists. In addition, during therapy, the realtime torques monitoring of each joint are display on the operation monitor, the patients can observe their performance from this real-time torque monitoring. This will make the patients know about their performance of training. Increasing of the training duration and the number of training sessions also improve the progress. However, the 2nd patient seem to have the constant condition due to not enough effort during training. The main point is that there is no side effect even through patients are not seriously perform trainings.

Four out of five subacute stroke patients have motor recovery of upper extremity after 2 weeks of conventional training plus using CUREs robot training. Recovery may due to either spontaneous or training, or both. Recovery is not enough to improve function. Total dose of training is too short. However, this study is mainly to observe the side effect for using this rehabilitation robot as shown in the Table 4 . This is the requirement of the institutional ethical committee. Now, we are performing the evaluation of the performance of curing of this rehabilitation robot. There are about 80 patients volunteer for the study. The results of the performance evaluation will be appeared in some medical journal. By the way, the performance evaluation is not required to qualify as a commercial product.

\section{Conclusion}

This article, CUREs was implemented with a healthy subjects and five subacute stroke patients. The experimental results showed that the active assistive control strategy with the CUREs can be used for stroke rehabilitation without any side effect based on the Fugl-Meyer Assessment Upper Extremity Scale (FMAUE). The results from the healthy subjects showed the trend of improvement of the motor recovery of upper extremity. And from the experimental results of the stroke patients showed that movement assistance from the exoskeleton robot has decreased within 2 weeks of the training. Even though the training period is rather short compare to the normal training period of the patient with severe condition, it is enough to confirm the 
safety of the system. And from the FMA-UE, there is no side effect for using the system. This is very important state of clinical trial. The experimental results also shown very promising that the stoke patients have motor recovery after training with the exoskeleton arm for the period of time.

\section{Acknowledgement}

This research is supported by National Research University Project, Office of Higher Education Commission (WCU-013-HR-57), The 100 $0^{\text {th }}$ Anniversary Chulalongkorn University Fund for Doctoral Scholarship and Regional Center of Robotics Technology, Chulalongkorn University. The authors declare that there is no conflict of interest regarding the publication of this paper.

\section{References}

[1] G. Kwakkel, R. C. Wagenaar, T.W. Koelman, G. J. Lankhorst, and J. C. Koetsier, "Effects of intensity of rehabilitation after stroke. A research synthesis," Stroke, vol. 28, no. 8, pp. 1550-1556, 1997.

[2] R. Teasell, J. Bitensky, K. Salter, and N. A. Bayona, "The role of timing and intensity of rehabilitation therapies," Top. Stroke Rehabil., vol. 12, no. 3, pp. 46-57, 2005.

[3] R. Riner, T. Nef, and G. Columbo, "Robot-aided neurorehabilitation for the upper extremities," Medical and Biological Engineering and Computing, vol. 43, pp. 2-10, 2005.

[4] M. Lotze, C. Braun, N. Birbaumer, S. Anders, and L. G. Cohen, "Motor learning elicited by voluntary drive," Brain, vol. 126, no. 4, pp. 866-872, 2003.

[5] D. J. Reinkensmeyer, L. E. Kahn, M. Averbuch, A. N. McKenna-Cole, B. D. Schmit, and W. Z. Rymer, "Understanding and treating arm movement impairment after chronic brain injury: Progress with the ARG Guides," J Rehabil Res Dev., vol. 37, no. 6, pp. 653-662, 2000.

[6] S. Masiero, A. Celia, G. Rosati, and M. Armani, "Robotic-assisted rehabilitation of the upper limb after acute stroke," Arch Phys Med Rebabil., vol. 88, pp. 142-9, 2007.

[7] N. Norouzi-Gheidari, P. S. Archambault, and J. Fung, "Effects of robot-assisted therapy on stroke rehabilitation in upper limb: Systematic review and meta-analysis of the literature," J. Rehabil. Res. Dev., vol. 49, pp. 479-96, 2012.

[8] P. Maciejasz, J. Eschweiler, K. Gerlach-Hahn, A. Jansen-Troy, and S. Leonhardt, "A survey on robotic devices for upper limb rehabilitation," Journal of Neuroengineering and Rehabilitation, vol. 11, no. 1, p. 3, 2014.

[9] N. Hogan, "Rehabilitation robotic: Performance-based progressive robot-assisted therapy," Autonomous Robot, vol. 15, no. 1, pp. 7-20, 2003.

[10] T. Nef, M. Guidali, and R. Riener, "ARMin III-arm therapy exoskeleton with an argonomic shoulder actuation," Applied Bionics and Biomechanics, vol. 6, no. 2, pp. 127-142, 2009.

[11] J. Rosen and J. C. Perry, "Upper limb powered exoskeleton," International Journal of Humanoid Robotics, vol. 4, no. 3, pp. 529-548, 2007.

[12] A. Sutapun and V. Sangveraphunsiri, "A 4-DOF upper limb exoskeleton for stroke rehabilitation: Kinematics mechanics and control," International of Mechanical Engineering and Robotics Research, vol. 4, no. 3, pp. 269-272, 2015.

[13] L. Marchal-Crespo and D. J. Reinkensmeyer, "Review of control strategies for robotic movement training after neurologic injury," Journal of Neuroengineering and Rehabilitation, vol. 6, no. 1, p. 20, 2009.

[14] H. Vallery, J. Veneman, E. V. Asseldonk, R. Ekkelenkamp, M. Buss, and H. Van Der Kooij, "Compliant actuation of rehabilitation robots," IEEE Robotic \& Automation Magazine, vol. 15, no. 3, pp. 60-69, 2008.

[15] L. L. Cai, A. J. Fong, C. K. Otoshi, Y. Liang, J. W. Burdick, R. R. Roy, and V. R. Edgerton, "Implications of assist-as-needed robotic step training after a complete spinal cord injury on intrinsic strategies of motor learning," Journal of Neuroscience, vol. 26, no. 41, pp. 10564-10568, 2006.

[16] N. Hogan, H. I. Krebs, B. Rohrer, J. J. Palazzolo, L. Dipietro, S. E. Fasoli, J. Stein, R. Hughes, W. R. Frontera, D. Lynch, and B. T. Volpe "Motions or muscles? Some behavioral factors underlying robotic assistance of motor recovery," J. Rehabil. Res. Dev., vol. 43, no. 5, pp. 605-618, 2006.

[17] M. Ferraro, J. J. Palazzolo, J. Krol, H. I. Krebs, N. Hogan, and B. T. Volpe, "Robot-aided sensorimotor arm training improves outcome in patients with chronic stroke," Neurology, vol. 61, no. 11, pp. 1604 $1607,2003$. 
[18] A. Sutapun and V. Sangveraphunsiri, "Dexterity measures for 4DOF exoskeleton robot," Applied Mechanics and Materials, vol. 619, pp. 214-218, 2014.

[19] W. Kitisomprayoonkul, P. Bhodhiassana, and V. Sangveraphunsiri, "Upper extremity training with CUREs robot in subacute stroke: A pilot study," in Converging Clinical and Engineering Research on Neurorehabilitaiton II. Springer, 2016, pp. 317-322.

[20] T. Nef, M. Miheli, and R. Riener, "ARMin: A robot for patient-cooperative arm therapy," Nedical and Biological Engineering and Computing, vol. 45, no. 9, pp. 887-900, 2007.

[21] J. L. Emken, S. J. Harkema, J. Beres-Jones, C. K. Ferreira, and D. J. Reinkensmeyer, "Feasibility of manual teach-and replay and continuous impedance shaping for robotic locomotor training following spinal cord injury," IEEE Transactions of Biomedical Engineering, vol. 55, pp. 322-334, 2008.

[22] J. J. Craig, Introduction to Robotics: Mechanics and Control, 3rd ed. USA: Pearson Education, 2005.

[23] S. B. Niku, Introduction to robotics: Analysis, control, Applications, 2nd ed. USA:_John Wiley \& Sons, 2011.

[24] H. Valley, H. Ekkelenkamp, V. D. Kooij, and M. Buss, "Passive and accurate torque control of series elastic actuators," in Proc. IEEE IROS, San Diego, CA, 2007, pp. 3534-3538.

[25] V. Sangveraphunsiri, K. Malithong, and S. Bongbunyong, "A control technique for a 6-DOF masterslave robot manipulator system for miniature task operation," presented at The 6th International Conference on Automotive Engineering (ICAE-6), Bangkok, Thailand, March 29-April 2, 2010.

[26] D. Nawakorn and V. Sangveraphunsiri, "Movable virtual wall for operation of master-slave manipulator arms," Applied Mechanics and Materials, vol. 415, pp.45-51, 2013.

[27] A. R. Fugl-Meyer, L. Jaasko, L. Leyman, S. Olsson, and S. Steglind, "The post-stroke hemiplegic patient. A method for evaluation of physical performance," Scan J Rehabil Med, vol. 7, no. 13-31, 1975.

[28] U. Phankaew, S. Tipchatyotin, P. Chantorn, K. Moungsunthorn, and S. Pianmanakit, "Interrater reliability of the Thai version Motor Assessment Scale for evaluation of upper extremity function in stroke patients," J Thai Rehabil Med, vol. 17, pp. 20-25, 2007.

[29] T. Flash and N. Hogan, "The coordination of arm movements: An experimentally confirmed mathematical model," Journal of Neuroscience, vol. 5, no. 7, pp. 1688-1703, 1985.

[30] M. Lotze, C. Braun, N. Birbauer, S. Ander, and L. G. Cohen, "Motor learning elicited by voluntary drive," Brain, vol. 126, no. 4, pp. 866-872, 2003.

[31] D. J. Reinkensmeyer, L. E. Kahn, M. Averbuch, A. N. McKenna-Cole, B. D. Schmit, and W. Z. Rymer, "Understanding and treating arm movement impairment after chronic brain injury: Progress with the ARM Guides," J Rehabil Res Dev, vol. 37, no. 6, pp. 653-662, 2000. 